\title{
A method for accurate detection of genomic microdeletions using real-time quantitative $\mathbf{P C R}$

\author{
Rosanna Weksberg ${ }^{\dagger 1,2}$, Simon Hughes ${ }^{\dagger 3}$, Laura Moldovan ${ }^{1}$, Anne S Bassett ${ }^{4}$, \\ Eva WC Chow ${ }^{4}$ and Jeremy A Squire*5
}

Address: ${ }^{1}$ Program in Genetics and Genomic Biology, The Research Institute, The Hospital for Sick Children, Toronto, Canada, ${ }^{2}$ Division of Clinical and Metabolic Genetics, Department of Paediatrics, The Hospital for Sick Children, Toronto, Canada, ${ }^{3}$ Queen Mary's School of Medicine and Dentistry, London, UK, ${ }^{4}$ Clinical Genetics Research Program, Centre for Addiction and Mental Health, and Department of Psychiatry, University of Toronto, Toronto, Canada and ${ }^{5}$ Ontario Cancer Institute and Department of Laboratory Medicine, Pathology and Medical Biophysics, University of Toronto, Toronto, Canada

Email: Rosanna Weksberg - rweksb@sickkids.ca; Simon Hughes - sih3@yahoo.com; Laura Moldovan - Laura.Moldovan@sickkids.ca; Anne S Bassett - Anne.Bassett@utoronto.ca; Eva WC Chow - Eva.Chow@utoronto.ca; Jeremy A Squire* - Jeremy.Squire@utoronto.ca

* Corresponding author †Equal contributors

\section{Published: 13 December 2005}

BMC Genomics 2005, 6:180 doi:10.1/86/|47|-2164-6-180
Received: 23 June 2005

Accepted: 13 December 2005

This article is available from: http://www.biomedcentral.com/I47I-2/64/6//80

(C) 2005 Weksberg et al; licensee BioMed Central Ltd.

This is an Open Access article distributed under the terms of the Creative Commons Attribution License (http://creativecommons.org/licenses/by/2.0), which permits unrestricted use, distribution, and reproduction in any medium, provided the original work is properly cited.

\begin{abstract}
Background: Quantitative Polymerase Chain Reaction (qPCR) is a well-established method for quantifying levels of gene expression, but has not been routinely applied to the detection of constitutional copy number alterations of human genomic DNA. Microdeletions or microduplications of the human genome are associated with a variety of genetic disorders. Although, clinical laboratories routinely use fluorescence in situ hybridization (FISH) to identify such cryptic genomic alterations, there remains a significant number of individuals in which constitutional genomic imbalance is suspected, based on clinical parameters, but cannot be readily detected using current cytogenetic techniques.

Results: In this study, a novel application for real-time QPCR is presented that can be used to reproducibly detect chromosomal microdeletions and microduplications. This approach was applied to DNA from a series of patient samples and controls to validate genomic copy number alteration at cytoband $22 \mathrm{ql}$ I. The study group comprised 12 patients with clinical symptoms of chromosome 22ql I deletion syndrome (22q I IDS), I patient trisomic for 22q II and 4 normal controls. 6 of the patients (group I) had known hemizygous deletions, as detected by standard diagnostic FISH, whilst the remaining 6 patients (group 2) were classified as 22qIIDS negative using the clinical FISH assay. Screening of the patients and controls with a set of 10 real time qPCR primers, spanning the 22qII.2-deleted region and flanking sequence, confirmed the FISH assay results for all patients with 100\% concordance. Moreover, this QPCR enabled a refinement of the region of deletion at $22 \mathrm{ql}$ I. Analysis of DNA from chromosome 22 trisomic sample demonstrated genomic duplication within $22 \mathrm{q} I \mathrm{l}$.

Conclusion: In this paper we present a qPCR approach for the detection of chromosomal microdeletions and microduplications. The strategic use of in silico modelling for $\mathrm{QPCR}$ primer design to avoid regions of repetitive DNA, whilst providing a level of genomic resolution greater than standard cytogenetic assays. The implementation of qPCR detection in clinical laboratories will address the need to replace complex, expensive and time consuming FISH screening to detect genomic microdeletions or duplications of clinical importance.
\end{abstract}




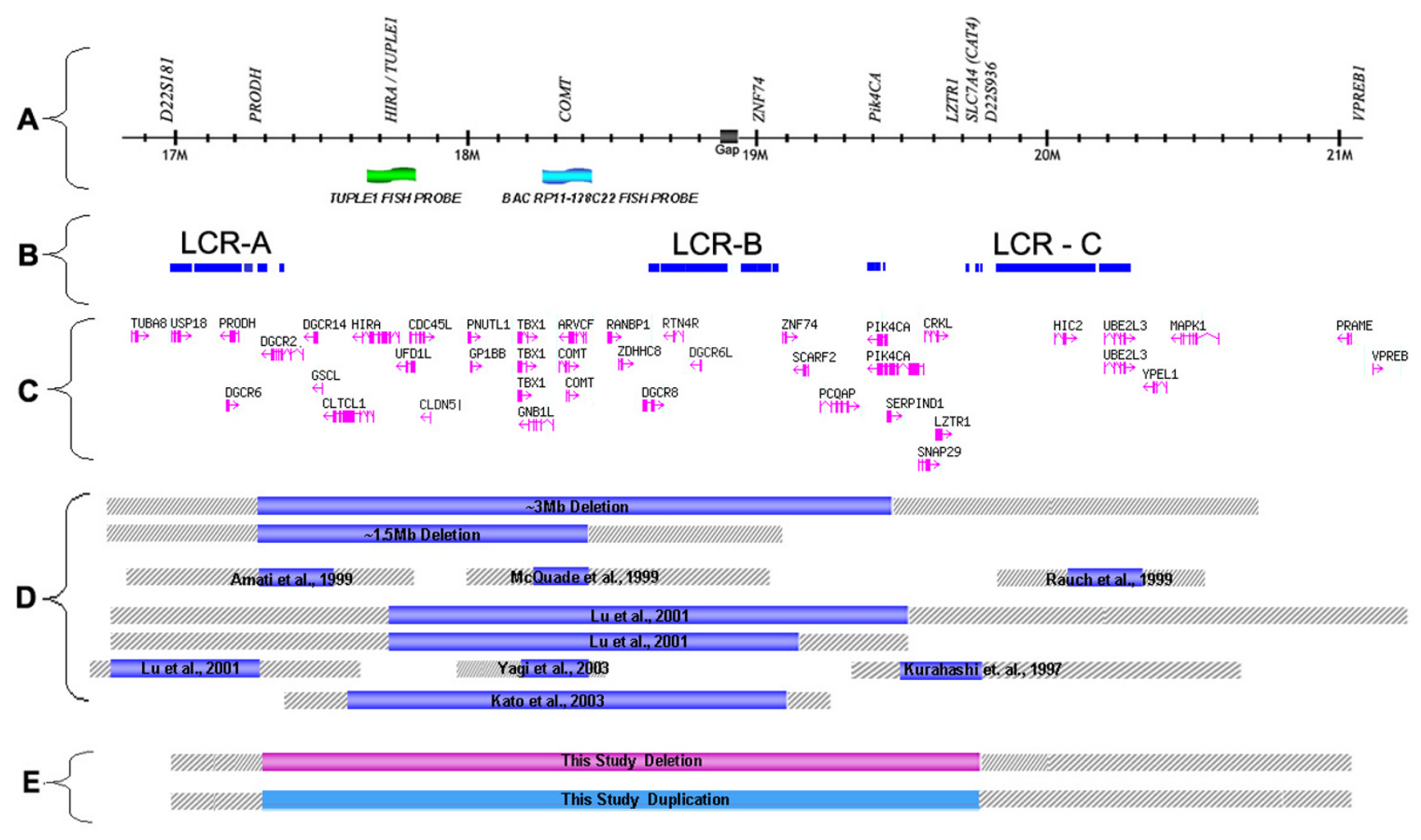

Figure I

Schematics of the 22q I I.2 region. Previously reported deletions and deletions identified from our study are shown. A) The 10 qPCR primers used to screen for hemizygous deletions, orientation is centromere to telomere. B) Known low copy number repeats or segmental duplications in 22q I I.2: LCR-A, LCR-B and LCR-D (Shaikh et al., 2000). C) Known genes [24]. D) Location of previously reported deletions in 22q I IDS patients. E) Locations of hemizygous deletions and duplications identified in this study. For $\mathrm{D}$ and $\mathrm{E}$, hashed ends represent regions of uncertainty regarding precise location of deletion breakpoints.

\section{Background}

Array comparative genomic [1-3] and FISH-based methods [4] have been widely used for the detection of DNA copy number changes. However, the resolution of commercially available DNA arrays can be too low to detect microdeletions or microduplications [5,6], whilst FISH is generally only useful when the regions of interest have been previously defined. Currently, DNA arrays providing full coverage of the human genome are not widely available and too expensive to diagnostically screen large numbers of patients. Moreover, the findings that are emerging from recent array comparative genomic hybridization studies indicate that significant validations of both controls and patient populations will be required to make phenotype-genotype interpretations in a clinical context. Similarly, standard FISH methods are time consuming, costly and suffer the significant limitation that some patients with uniquely localized microdeletions or duplications may yield normal clinical FISH findings because the probe set used does not map precisely to the entire region of deletions/duplication.

In this study we have chosen to validate the use of qPCR technology for detection of microdeletions or microduplications using Velocardiofacial Syndrome (VCFS) or chromosome 22q11 deletion syndrome (22q11DS) as a test model. The frequency of the causative deletion for 22q11DS in the general population is 1 in 3000 live births, making it one of the most common constitutional genomic alterations found in humans [7]. 22q11DS is suspected in individuals with characteristic clinical findings and is confirmed in most cases by detection of a submicroscopic deletion using FISH.

The currently accepted clinical laboratory assay for 22q11DS uses the TUPLE1 FISH probe, which is located within a typically deleted region of approximately $3 \mathrm{Mb}$. 
Table I: Sequence and parameters of the reference and $22 \mathrm{q}$ I .2 test primer sets. Ten sets of primers were designed from within regions of unique sequence on $22 \mathrm{q}$ I I.2 using Primer Express v2.0. In addition, two sets of reference primer for G6PDH and HEM3 were also designed to allow for data correction.

\begin{tabular}{|c|c|c|c|c|c|c|c|c|c|}
\hline Primer Sets & No. & Primer Name & Primer Sequence & Chr. & $\begin{array}{l}\text { Genomic Location } \\
\text { of Amplicon }\end{array}$ & $\begin{array}{l}\text { Amplicon } \\
\text { Size (bp) }\end{array}$ & $\begin{array}{l}\text { Cycle } \\
\text { Treshold }\end{array}$ & Slope & $\mathbf{R 2}$ \\
\hline \multirow[t]{4}{*}{ Reference } & $\mathbf{I}$ & G6PDH-F & TCTTCATCACCACAGAGAACTTGC & 1 & $9033513-9033733$ & 221 & 0.2 & -3.2968 & 0.9980 \\
\hline & & G6PDH-R & GACCTGGAAGTCACTGGGCA & & & & & & \\
\hline & 2 & HEM3-F & TGCACGGCAGCTTAACGAT & 11 & $118501618-118501818$ & 202 & 0.2 & -3.3998 & 0.9981 \\
\hline & & HEM3-R & AGGCAAGGCAGTCATCAAGG & & & & & & \\
\hline \multirow[t]{20}{*}{ Test } & $\mathbf{I}$ & $\mathrm{D} 22 \mathrm{SI} / 8 \mathrm{I}-\mathrm{F}$ & CAGCTCCCAAGTCTTTCCAGC & 22 & $16968759-16968859$ & 101 & 0.2 & $-3.4|4|$ & 0.9986 \\
\hline & & $\mathrm{D} 22 \mathrm{~S} 182-\mathrm{R}$ & CCAGGGTAGGAAACAGGTCGA & & & & & & \\
\hline & 2 & PRODH-F & GGGAAAGGAGAGTTCAGGCAG & 22 & $17293217-17293317$ & 101 & 0.2 & -3.5102 & 0.9992 \\
\hline & & PRODH-R & GCTTGTTGAATAGCCTCTGTCCTAG & & & & & & \\
\hline & 3 & TUPLEI-F & GGCAAGTGCAATATTCATGTGGT & 22 & $17763150-17763250$ & 101 & 0.2 & -3.2564 & 0.9996 \\
\hline & & TUPLEI-R & TCCTACACGCCTGACAAAGCT & & & & & & \\
\hline & 4 & COMT-F & GTGCTACTGGCTGACAACGTGAT & 22 & $18330640-18330739$ & 100 & 0.2 & -3.5876 & 0.9928 \\
\hline & & COMT-R & GGAACGATTGGTAGTGTGTGCA & & & & & & \\
\hline & 5 & ZNF74-F & TGGCCTCCTGCTTCTTTCTTC & 22 & $19073892-19073992$ & 101 & 0.2 & -3.2263 & 0.9960 \\
\hline & & ZNF74-R & CAGACACTCCAATTCATGACGAA & & & & & & \\
\hline & 6 & PIK4CA-F & ATGCTTGTGCGACGCAGAC & 22 & $19429805-19429905$ & 101 & 0.06 & -3.0238 & 0.9958 \\
\hline & & PIK4CA-R & CCTCAGCCATGTTGACTCAGC & & & & & & \\
\hline & 7 & LZTRI-F & TCATCATGGATGTGTACAAACTGG & 22 & $19673422-19673524$ & 103 & 0.2 & -3.2416 & 0.9977 \\
\hline & & LZTRI-R & AGCACGTTCTGCAGGTCCAC & & & & & & \\
\hline & 8 & CAT4-F & TACCTGGGCTTCTTGGATGG & 22 & | $9708684-19708784$ & 101 & 0.2 & -3.4394 & 0.9986 \\
\hline & & CAT4-R & AAGACAAGCACGCAGCCTATG & & & & & & \\
\hline & 9 & D22S936-F & TGGCAGCCAGTTTAGTATTCTGC & 22 & $|97773| 4-197774 \mid 4$ & 101 & 0.2 & -3.1972 & 0.9960 \\
\hline & & D22S936-R & TTGTAATCAAGTCCCGCCACT & & & & & & \\
\hline & 10 & VPREBI-F & CGACCATGACATCGGTGTGT & 22 & 20924000-20924102 & 103 & 0.2 & -3.2923 & 0.9972 \\
\hline & & VPREBI-R & CTGGCTCTTGTCTGATTGTGAGA & & & & & & \\
\hline
\end{tabular}


Although this assay can detect the majority of affected patients (85-90\%), many patients with phenotypic features of 22q11DS have no deletion detectable by FISH testing. As a consequence these patients will go undiagnosed due to the presence of atypical deletions that map outside the area covered by the TUPLE1 probe [8-12]. In addition, there have been reports of individuals with some features of 22q11DS with microduplications of 22q11.2 [13]. Unfortunately, clinical FISH assays are not usually capable of detecting such duplications, so alternative methods, such as FISH analysis of interphase nuclei, are required [14]. However, such techniques require advanced optical instrumentation, presently only used in specialized research laboratories. Alternative molecular technologies that could potentially be used to screen deletions in the diagnose of 22q11DS is multiplex ligationdependent probe amplification (MLPA) [15] or microsatellites marker analysis [10].

The novel approach validated in this study utilized qPCR rather than FISH to detect copy number alterations (microdeletions and microduplications) in patient DNA. This approach had several advantages. Primers were selected within regions of unique sequence utilizing publicly available sequence databases [16-24]. This method can allow for the production of a high-resolution map of any region of interest; the 22q11DS region is used in this example. The qPCR technique provides a quantitative measurement of DNA copy number and accurately characterizing chromosomal breakpoints. This method will therefore permit the identification of individuals who would otherwise go undetected by the currently available clinical FISH methods. In addition, qPCR provides greater flexibility and adaptability, whilst being less technically challenging than FISH, thus making it more appropriate for use in a large number of laboratories. Furthermore, the qPCR technique only takes a fraction of the time usually required for FISH, which allows for multiple samples and multiple primer sets to be studied in parallel, using convenient and cost effective high throughput analysis. The method described in this paper has been evaluated using patient and controls samples with known copy number changes on $22 \mathrm{q} 11$. The approach can be readily adapted for molecular diagnostics of any region of the genome suffering recurrent constitutional genomic deletion or duplication.

\section{Results}

\section{Fish results}

The twelve patients utilized in this study have previously been analysed in a clinical laboratory by FISH using the TUPLE 1 probe. Using this test, six of the patients (group 1) were identified as $22 \mathrm{q} 11$ deletion positive whilst the other six (group 2) and the four controls showed normal cytogenetic results. The DNA sample known to have three copies of 22q11 (trisomy for chromosome 22) had been previously analysed by FISH [25].

\section{Primer design}

The most critical factor for successful detection of microalterations using qPCR was primer design. To guarantee optimal primer design a high-resolution 22q11.2 physical map was constructed using information available from published reports [8-12,26-34] and online databases and repositories [16-24]. This allowed for the identification of unique sequences within the 22q11DS affected region whilst also avoiding the complex repetitive regions. Figure 1 shows a schematic representation of the 22q11.2 region studied in this work, the location of previously reported deletions and deletions identified from our study are shown as are the location of primers, repeat sequences, known genes and pseudogenes.

Sequences were selected to lie, mostly, within exonic and/ or intervening regions from known or putative genes: UniSTS marker D22S181 (UniSTS is a comprehensive database of sequence tagged sites) [35], Proline Dehydrogenase/Proline Oxidase (PRODH), TUP-like enhancer of SPLIT 1 (TUPLE1), Catechol-O-Methyltransferase (COMT), Zinc Finger Protein 74 (ZNF74), Phosphatidylinositol 4-kinase (PIK4CA), Leucine-zipper-like Transcriptional Regulator 1 (LZTR1), Cationic Amino acid Transporter-4 (CAT4), D22S936 and Similar to Pre-B lymphocyte gene 1 (VPREB1). The selected sequences were aligned against the human genome using the BLAT program [36] to ensure that only contiguous sequences with $100 \%$ homology to one unique location were selected. A single primer set was then designed from each of these unique sequences using the Primer Express v 2.0 program (Applied Biosystems). We designed reference primers for each of the "housekeeping genes" Glucose-6-Phosphate Dehydrogenase (G6PDH) and Hydroxymethylbilane Synthase (HEM3) which were selected using published guidelines [37]; moreover we insured that they were single copy genomic sequences according to the BLAT alignment [38], in addition agarose gel visualization confirmed a single band of the expected size. All primer sequences are shown in Table 1. The use of reference primers was to control for varying input amounts of DNA from each separate patient. Thus any differences in the qPCR values obtained for test primers/markers would correspond to differences in the amount of the primers target sequence.

\section{Optimization process}

Primer concentrations were optimised over a range of final concentrations, $100 \mathrm{nM}$ to $900 \mathrm{nM}$ at $100 \mathrm{nM}$ intervals. The optimal concentration was that which obtained the lowest threshold cycle $\left(\mathrm{C}_{\mathrm{t}}\right)$ and maximum $\Delta \mathrm{R}_{\mathrm{n}}$ while minimizing non-specific amplification. The results indicated that for all of the 22q11.2 primer sets the optimal 
Table 2: Fold copy number change ( $\Delta K C_{t}$ ) for the $1222 q$ I IDS patients, $22 q$ I I Duplication and 4 controls. $\Delta K C_{t}$ values of $0 \pm 0.35$ indicate an equal ratio of the target and reference, which corresponds to no loss and therefore no genetic abnormality, values of $-I \pm$ 0.35 indicate loss of one copy (microdeletion), whilst values of $I \pm 0.35$ (seen for the trisomy 22 patient) indicate gain of one copy (microduplication).

\begin{tabular}{|c|c|c|c|c|c|c|c|c|c|c|c|}
\hline \multirow[t]{3}{*}{ Sample Types } & \multirow[t]{3}{*}{ Samples } & \multicolumn{10}{|c|}{ qPCR Primers of the 22q I I.2 DS Region } \\
\hline & & D22SI8I & PRODH & TUPLEI & COMT & ZNF74 & PIK4 & LZTRI & CAT4 & D22S936 & VPREB I \\
\hline & & & & & Fold co & py number & change $(L$ & $K C t)$ & & & \\
\hline \multirow{4}{*}{ Normal Controls } & $\mathbf{I}$ & -0.1601 & 0.2039 & 0.1716 & 0.1273 & 0.0744 & 0.1097 & -0.1598 & 0.0175 & -0.2195 & 0.1782 \\
\hline & 2 & 0.0881 & -0.0115 & -0.0703 & -0.0045 & -0.0744 & $-0.142 \mid$ & 0.0538 & -0.1002 & -0.0327 & 0.1738 \\
\hline & 3 & -0.0802 & -0.1322 & -0.0202 & 0.0471 & 0.1633 & 0.0211 & 0.1497 & -0.1193 & 0.1580 & -0.1393 \\
\hline & 4 & -0.2727 & -0.0602 & -0.0812 & -0.1700 & 0.2048 & 0.0113 & -0.2035 & -0.1173 & -0.1508 & -0.2128 \\
\hline \multirow{6}{*}{$\begin{array}{c}\text { 22q I IDS Tuple I } \\
\text { Deleted }\end{array}$} & I & -0.2006 & -1.2337 & -1.2173 & -0.9261 & -1.2366 & -1.1903 & -0.9009 & -1.1467 & -1.1305 & 0.0140 \\
\hline & 2 & 0.1987 & -1.1857 & -1.2806 & -1.1102 & -0.8132 & -0.9187 & -1.2182 & -0.8644 & -1.1021 & -0.0667 \\
\hline & 3 & -0.0035 & -1.2003 & $-|.167|$ & -1.2660 & -1.0857 & -1.2183 & -0.8808 & -1.1124 & -1.3388 & -0.3315 \\
\hline & 4 & -0.0387 & -1.2372 & -1.2534 & -1.2976 & -0.9337 & -1.1139 & -1.1951 & -0.9513 & -0.9711 & 0.0759 \\
\hline & 5 & -0.2601 & -0.9173 & -1.1538 & -0.9330 & -0.9639 & -0.9715 & -1.2522 & -1.0367 & -1.1721 & 0.1191 \\
\hline & 6 & 0.0158 & $-|.054|$ & -1.1710 & -0.8799 & -0.9787 & -0.9763 & -1.0172 & -0.9620 & -1.3164 & 0.1496 \\
\hline \multirow{6}{*}{$\begin{array}{l}\text { 22q I IDS Tuple I } \\
\text { Not-Deleted }\end{array}$} & I & 0.0670 & -0.1259 & 0.0717 & -0.1508 & 0.2653 & $-0.024 I$ & -0.1144 & -0.1205 & 0.0881 & 0.0140 \\
\hline & 2 & -0.0325 & -0.0797 & -0.1033 & -0.1055 & 0.1414 & -0.0507 & -0.1921 & -0.1158 & -0.0905 & -0.0667 \\
\hline & 3 & -0.1568 & -0.0003 & 0.1309 & -0.1154 & 0.1521 & -0.0615 & -0.0400 & 0.1025 & 0.1660 & -0.3315 \\
\hline & 4 & -0.0737 & -0.1425 & 0.0046 & 0.0205 & 0.2222 & -0.0246 & -0.1283 & 0.1719 & 0.1093 & 0.0759 \\
\hline & 5 & 0.1166 & 0.0283 & -0.0596 & 0.1068 & 0.1951 & -0.0478 & -0.1887 & 0.1936 & 0.0282 & 0.1191 \\
\hline & 6 & -0.0508 & 0.1578 & 0.0755 & 0.0324 & 0.1366 & -0.0066 & 0.0569 & 0.1882 & 0.0900 & 0.1496 \\
\hline \multicolumn{2}{|c|}{ 22q I I Duplication Sample } & -0.0124 & 1.1855 & 0.9201 & 0.9303 & 1.0121 & 0.9377 & 1.0648 & 0.9637 & 1.0250 & -0.1863 \\
\hline
\end{tabular}

final primer concentration was $800 \mathrm{nM}$, whilst for the G6PDH and HEM3 primer sets this value was $400 \mathrm{nM}$. The specificity of amplification for each qPCR product was confirmed by determining that the melting curve, showed a single dissociation peak corresponding to the melting temperature of the analysed amplicons (See Additional File 3, Figure 2 b. Dissociation Curve for PRODH 14 DNA samples).

To allow for comparisons between each primer sets all had to amplify with comparable efficiency. This was assessed by analysis of the slopes from the standard curves, which were generated using a $\log _{10}$ dilution series of input genomic DNA (range $10^{2} \mathrm{nM}$ to $10^{-2} \mathrm{nM}$ ). If all conditions are optimal and reactions are $100 \%$ efficient, it will take approximately 3.32 cycles for ten fold amplification $\left(\log _{2}\right.$ of $\left.10=3.321928\right)$ of product, a value that is equal to the slope of the standard curve. This translates to 1 cycle to copy 1 molecule into 2 ; a second cycle to copy 2 molecules into 4 ; a third cycle to copy 4 into 8 and 0.32 cycles to copy 8 into 10 . There was a linear relationship between the amount of input DNA and the threshold cycle $\left(\mathrm{C}_{\mathrm{t}}\right)$ values for the various reactions. Regression analyses of the $C_{t}$ values generated by the $\log _{10}$ dilution series gave $\mathrm{R}^{2}$ values for all reactions in excess of 0.99 (Table 1 ).
At the optimal primer concentrations all of the primer sets gave slope values of $3.32 \pm 0.25$, indicating that the reactions were occurring with similar efficiencies. Following primer optimisation a baseline $\mathrm{C}_{t}$ was identified for each primer set, which was used when analysing subsequent data $\left(\mathrm{R}^{2}\right.$, slope and threshold cycle values are shown in Table 1).

\section{Data correction/normalization}

To control for differences in sampling, DNA preparation, reaction efficiency (the varying PCR efficiencies between patient samples and the calibrator) [39] and other variables such as the $C_{t}$ values for each primer pair [40] from $22 q 11.2$ were corrected/normalized using the $C_{t}$ value of the G6PDH and HEM3 products for the same sample. Although the input of template was standardised at $10 \mathrm{ng}$ of DNA, the $\mathrm{C}_{\mathrm{t}}$ values for the housekeeping genes differed slightly from patient to patient and from group to group (Table 3), thus demonstrating the need for correction of the raw data prior to further analysis.

Correction was performed using a method described by Moody et al. (2000) [41]. Once the corrected $C_{t}$ values $\left(\mathrm{KC}_{\mathrm{t}}\right)$ for each of the test markers had been determined it was then possible to identify fold copy number change 
Table 3: Example of uncorrected $\mathrm{Ct}$ values characteristic to the reference (housekeeping) primer G6PDH. Exemplification of the uncorrected $\mathrm{Ct}$ (average of three replicates) values characteristic to the reference (housekeeping) primer G6PDH of the DNA samples under study. Although starting concentration of DNA for all samples was $10 \mathrm{ng}$ the $C_{t}$ values for the housekeeping genes differed slightly from patient to patient and from group to group.

\begin{tabular}{ccc}
\hline Samples & & G6PDH Ct value \\
\hline Control & $\mathbf{I}$ & 21.2196 \\
& $\mathbf{2}$ & 21.1595 \\
& $\mathbf{3}$ & 23.0891 \\
& $\mathbf{4}$ & 21.3748 \\
\hline Group I & $\mathbf{I}$ & 19.8799 \\
& $\mathbf{2}$ & 21.2899 \\
& $\mathbf{3}$ & 20.5799 \\
& $\mathbf{4}$ & 20.7859 \\
& $\mathbf{5}$ & 21.4781 \\
& $\mathbf{6}$ & 19.5612 \\
\hline Group 2 & $\mathbf{1}$ & 20.3756 \\
& $\mathbf{2}$ & 21.1237 \\
& $\mathbf{3}$ & 21.1544 \\
& $\mathbf{4}$ & 21.1484 \\
& $\mathbf{5}$ & 20.5768 \\
& $\mathbf{6}$ & 19.9122 \\
\hline \multirow{2}{*}{ 22qDuplication } & $\mathbf{1}$ & 21.3409 \\
\hline
\end{tabular}

$\left(\Delta \mathrm{KC}_{\mathrm{t}}\right)$ for each of the markers from the 22q11.2 region, using a formula described by Sijben et al. (2003) [42]. In the context of 22q11DS this approach calculates a ratio; by comparing the $C_{t}$ value obtained for each primer pair between the normal (control) DNA samples and the patient (affected) DNA samples. This value is then translated into fold changes (copy number gain or loss) per sample. For all calculations please refer to the Methods section.

\section{qPCR data}

A summary of the qPCR results is presented in Table 2; light grey shading denotes loss, whilst dark grey shading denotes gain. We obtained $\Delta \mathrm{KC}_{\mathrm{t}}$ values of either $0 \pm 0.35$ indicating an equal ratio of the target and reference, which corresponds to no loss and therefore no genetic abnormality, or $-1 \pm 0.35$, indicating loss of one copy (microdeletion), for the affected samples. For the trisomy 22 patient we obtained a ratio of $1 \pm 0.35$, indicating gain of one copy. Each experiment was performed in triplicate, with replicates performed on different days. The interassay (same assay repeated on different days) $\mathrm{C}_{t}$ variation and the intra-assay $\mathrm{C}_{\mathrm{t}}$ variation (the triplicates) was less than or equal to \pm 0.35 cycles.

\section{Discussion}

In our analysis we have used a series of 12 qPCR primer sets to analyze twelve patients with clinical symptoms of 22q11DS and four controls. Ten of the primer sets (test primers) amplify markers localized within and around the chromosome 22q11.2-deleted region (D22S181, PRODH, TUPLE1, COMT, ZNF74, PIK4CA, LZTR1, CAT4, D22S936 and VPREB1) and two amplify "housekeeping" genes/markers (reference primers) (G6PDH and HEM3). The 22q11DS locus contains approximately 50 genes or pseudogenes and is characterized by an unusual genomic architecture comprising a large polymorphic chromosome-specific low copy repeats (Figure 1)[34]. The repetitive nature of this region of the genome is thought to increase the frequency of deletions and duplication events associated with clinical disease. The repetitive nature of the region of 22q11.2 under study required bioinformatics to identify unique regions for primer design.

In the context of 22q11DS we were trying to discriminate between 2 copies of a product (normal) versus 1 copy of the same product (deleted). In order to do this we had to implement data correction [41] to control for variations such as the input DNA concentration or reaction efficiencies [39]. To perform the correction we used the reference genes. The copy number of the reference genes will be the same in all of the samples under investigation. Any variation in copy number for the reference between samples will be the result of differences in initial template concentration, as long as the same DNA is sampled for both the control and target primers. Once the $\mathrm{C}_{\mathrm{t}}$ values for the reference were determined for each sample, it was then possible to use these to correct the values of the test markers for variation in initial template concentration. This correction permitted determination of copy number differences between the samples under investigation.

Individuals who had previously shown a deletion of TUPLE1 by FISH also showed deletion by qPCR (group 1). Our data demonstrates that the $\Delta \mathrm{KC}_{\mathrm{t}}$ values, $-1 \pm 0.35$, for the primers PRODH, TUPLE1, COMT, ZNF74, PIK4CA, LZTR1, CAT4 and D22S936 are indicative of deletion (Table 2). A finding that is in 100\% concordance with the FISH results for the TUPLE1 probe. The region of deletion spanning PRODH, to D22S936, represents an interval of 2,502,410 base pairs (bp). Furthermore, the implementation of qPCR has allowed for the identification of breakpoint within the 22q11.2 region. The markers D22S181 and VPREB1 show values indicative of no deletion $(0 \pm 0.35)$, suggesting that the proximal deletion breakpoint occurs between the markers D22S181 and PRODH and the distal breakpoint between D22S936 and VPREB1 (see Table 2 and Figure 1). 
The individuals that did not demonstrate loss using the TUPLE1 FISH probe were also deletion negative by qPCR, showing $\Delta \mathrm{KC}_{\mathrm{t}}$ values similar to the normal controls $(0 \pm$ 0.35 ) thus indicative of no loss. For the 22q11 trisomy sample the qPCR results showed $\Delta \mathrm{KC}_{\mathrm{t}}$ values indicative of duplication $(1 \pm 0.35)$ for PRODH, TUPLE1, COMT, ZNF74, PIK4CA, LZTR1, CAT4 and D22S936. The markers D22S181 and VPREB1 again showed values indicative of no copy number change $(0 \pm 0.35)$.

To accurately calculate fold changes, to the high precision required for genomic DNA quantitation, the real-time data analysis used here makes the most of the existing methods. The slope values determined from the standard curve allows for correction for variations in the primers efficiency and reaction kinetics, whilst the relative abundance ratio, calculated after the samples are normalized using the reference genes, allows for determination of gene/marker copy number. This rationale reduces, as much as possible, the unavoidable approximations introduced with any method of data interpretation.

The use of qPCR to detect and refine copy number differences in patients suffering from 22q11DS provides a further novel application for qPCR methodology. When used in a research setting, this type of analysis has proven to be very useful when comparing levels of a transcript or genomic markers $[43,44]$ between different groups, and has been widely used in the study of human malignancies. However, such an approach has rarely been applied in a clinical setting to study constitutional deletions. The advantage of this technique over standard FISH assays is that qPCR provides a quantitative measurement of DNA copy number.

\section{Conclusion}

Here we demonstrate the application of a robust, fast and accurate real-time quantitative PCR based assay using SYBR ${ }^{\circledR}$ Green I dye, that is capable of screening for copynumber alterations in genomic DNA.

Although qPCR detection methods have previously been used in 22q11.2 deletion analysis [45-47], these reports have only used a small number of primers/markers and have not been able to refine the region of deletion as has been done here. The utility of the approach outlined in this paper is the ease with which one can increase resolution by increasing the number of primers in the $22 \mathrm{q} 11$ deleted region thus facilitating accurate mapping of deletion breakpoints. The fine structure of qPCR mapping of deletions will reveal important clues into the mechanism by which the deletion occurs and thus will offer insights into the "at risk" factors predictive of deletions or other rearrangements.
The implementation of qPCR for genomic copy number profiling will provide a valuable tool for detection of atypical microdeletions and/or microduplications in individuals who go undiagnosed by the current available FISH methods. Such data will be useful for phenotype correlation studies. In addition, this methodology has the advantage of providing greater flexibility and adaptability than the currently available cytogenetic methods and will be beneficial in molecular classification and diagnosis.

\section{Methods}

\section{Primer design}

Primers (Table 1) were designed using Primer Express v2.0 (Applied Biosystems). The parameters for primer design were as follows; amplicons of $100-250 \mathrm{bp}$ with a penalty score no higher than 10-12; primer melting temperature $\left(T_{\mathrm{m}}\right)$ range $58^{\circ} \mathrm{C}$ to $60^{\circ} \mathrm{C}$; primer length range 18 to 25 bp, optimal $20 \mathrm{bp}$; primer $\mathrm{G} / \mathrm{C}$ content range $20 \%$ to $60 \%$; amplicon maximum $T_{\mathrm{m}}$ of $85^{\circ} \mathrm{C}$. Runs of more than three identical nucleotides were avoided as polyG or polyC stretches can promote non-specific annealing, whilst runs of polyA and polyT can potentially open up stretches of the primer-template complex. Primers were required to be free of self-complementary sequence in order to avoid hairpin loop formation. In addition, no more than two $\mathrm{G}$ and/or $\mathrm{C}$ bases were permitted in the last five nucleotides at the 3' end to avoid GC clamp formation. Minimal deviation from the parameters was allowed only when there were no other options due to the complex nature of the 22q11.2 sequence. Primer and amplicon sequences were compared to the human genome using the BLAT program to guarantee that they showed $100 \%$ homology to only the sequence from which they were designed and also to guarantee that the forward and reverse primers were free of single nucleotide polymorphisms.

\section{Isolation of DNA}

The DNA used in this study was obtained from the lymphocytes of four normal controls and 12 patients displaying clinical features of 22q11DS using phenol-chloroform extraction [48]. DNA was quality tested. Suitable sample for the qPCR reactions were of high molecular weight (unsheared band of undigested DNA visible on a $0.5 \%$ agarose gel) and as clean as possible (an OD 260/280 ranging from 1.8 to 2.0 ).

\section{Reaction conditions}

Reactions were performed using SYBR Green I PCR Master Mix (Applied Biosystems), which includes the internal reference (ROX). Each qPCR reaction comprised $12.5 \mu \mathrm{l} 2 \times$ SYBR Green PCR Master Mix, forward and reverse primer at optimized concentrations of $800 \mathrm{nM}$ (final concentration) for the 22q11.2 test primers and $400 \mathrm{nM}$ (final concentration) for the reference primers, $10 \mathrm{ng} / \mu \mathrm{l}$ genomic 
DNA template and sterile water up to a final volume of 25 $\mu \mathrm{l}$. The qPCR reactions were performed using the ABI Prism 7900 high-throughput sequence detection system. The reaction profile was: initial step, $50^{\circ} \mathrm{C}$ for $2 \mathrm{~min}$, denaturation, $95^{\circ} \mathrm{C}$ for $10 \mathrm{~min}$, then 40 cycles of denaturing at $95^{\circ} \mathrm{C}$ for $15 \mathrm{sec}$ and combined annealing and extension at $60^{\circ} \mathrm{C}$ for $60 \mathrm{sec}$.

\section{Generating the standard curve}

To generate standard curves for the selected primers and the reference primers a $\log _{10}$ dilution series of genomic DNA was prepared at concentrations ranging from $10^{2} \mathrm{nM}$ to $10^{-2} \mathrm{nM}$. Each dilution was tested in triplicate. When analyzed by qPCR, the dilution series produced a set of standard curves, which were used to calculate the slope value with the aid of the SDS software version 2.1 Applied Biosystems (values are shown in Table 1). See Additional File 3, Figure 2 e. and $\mathrm{f}$. for an example of SDS output report of standard and amplification plots.

\section{DNA quantification data analysis}

Each qPCR experiment contained triplicates of the notemplate-controls and patient samples for all of the primers tested. On the same reaction plate all DNA samples were tested with the test and reference primers. When any particular sample was being tested, the qPCR using reference primers and that sample were always included on the reaction plate. Each experiment was performed in triplicate, with replicates being performed on different days. Quantification was based on the increased fluorescence, which was measured and recorded using the ABI Prism 7900 sequence detection system and associated SDS software version 2.1 (Applied Biosystems). Results were expressed in terms of the threshold cycle value $\left(C_{t} ;\right.$ the cycle at which the change in fluorescence for the SYBR dye passes a significance threshold). The threshold values are shown in Table 1. The output of the results was exported in tab-delimited text file format. Further calculations were performed using Microsoft Excel. PCR products were resolved by agarose gel electrophoresis to confirm the presence of a single band of the expected size (See Additional File 3, Figure 2 a. showing SDS output of amplification plot for 14 DNA samples for the PRODH and G6PDH primer sets along with images of corresponding gel bands c. and d.).

\section{Data normalization}

The qPCR data was normalized adapting a method devised by Moody et al. (2000) [41] and also described by Sijben et al. (2003) [42]. (See Additional File 1: Derivation of the formula)

$$
K C_{t i}=\left(\frac{A C_{t R}-C_{t R i}}{S_{R}}\right) \times S_{T}+C_{t T i}
$$

Where:

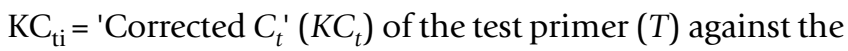
reference $(R)$

$\mathrm{AC}_{\mathrm{tR}}=$ the Average $\mathrm{C}_{\mathrm{t}}$ value for Reference primer set for all the samples included in one qPCR run (control and patient).

$\mathrm{C}_{\mathrm{tRi}}=\mathrm{Ct}$ value for Reference primer set for the sample to be corrected.

$S_{R}=$ slope value (from the standard curve) for reference primer set.

$\mathrm{S}_{\mathrm{T}}=$ slope value (from the standard curve) for test primer set.

$\mathrm{C}_{\mathrm{tTi}}=\mathrm{C}_{\mathrm{t}}$ value for test primer set.

\section{Copy number calculation}

Fold copy number $\left(\Delta \mathrm{KC}_{\mathrm{t}}\right)$ change for each of the markers from the 22q11.2 region, was obtained using the formula:

$\Delta K C_{t}=K C_{t / \text { control }}-K C_{t / \text { affected }}$

$\Delta \mathrm{KC}_{\mathrm{t}}=$ fold change (copy number gain or loss)

$\mathrm{KC}_{\mathrm{t} / \mathrm{control}}=$ "Corrected $\mathrm{C}_{\mathrm{t}}$ " of the test primer for the control samples.

$\mathrm{KC}_{\mathrm{t} / \text { affected }}=$ "Corrected $\mathrm{C}_{\mathrm{t}}$ " of the test primer for the affected sample.

(Of note when multiple controls were used in the same reaction run the $\mathrm{KC}_{\mathrm{t} / \mathrm{control}}$ was obtained by averaging all the controls'

"Corrected $\mathrm{C}_{\mathrm{t}}{ }^{\prime}-\mathrm{KC}_{\mathrm{t} / \mathrm{control}} \mathrm{s}$ ). (See Additional File 2: Working example)

\section{Abbreviations}

qPCR - Real time Quantitative Polymerase Chain Reaction

G6PDH - Glucose-6-Phosphate Dehydrogenase

Reference (R) - refers to the sequence we use to standardize against - the sequence, which we expect to remain unchanged in all the DNA samples (patient and control), and is the equivalent of the housekeeping gene use in expression studies. In our experiments we use G6PDH and HEM3. 
Target $(\mathrm{T})$ - refers to the sequence originating from the region of interest which we test and which we expect to find modifications (deletion or duplication).

\section{Authors' contributions}

JS and RW conceived the study, and participated in its design, coordination and final writing; $\mathrm{SH}$ resourced the mathematical model and the methodology, revised the data and the manuscript for publication; LM developed the required bioinformatics, carried out the molecular genetics study, performed the data interpretation adapting the mathematical model, additional materials and, together with SH drafted the manuscript; ASB and EWCC contributed microdeletion cell lines, pertinent clinical information and contributed to overall study design; all authors revised and approved the final manuscript.

\section{Additional material}

\section{Additional File 3}

Figure 2 (Fig. 2) Example of SDS output report and agarose gel for samples run on real-time qPCR. a. Amplification plot for PRODH (14 DNA samples) and G6PDH (14 DNA samples). $b$. Dissociation Curve PRODH -14 DNA samples. c. and d. Agarose gel electrophoresis images showing unique bands for 14 DNA samples qPCR products corresponding to $101 \mathrm{bp}$ fragment (PRODH primer set) and respectively $202 \mathrm{bp}-$ G6PDH. e. COMT Standard Curve Plot and f. Amplification Plot for standard dilutions.

Click here for file

[http://www.biomedcentral.com/content/supplementary/14712164-6-180-S3.pdf]

\section{Additional File 1}

MS Word 2000 document demonstrating the mathematical derivation of the formula for copy number transition (fold change).

Click here for file

[http://www.biomedcentral.com/content/supplementary/14712164-6-180-S1.doc]

\section{Additional File 2}

MS Word 2000 document describing the mathematical formula applied to a practical example (working example) where fold change is calculated from the Ct values of SDS output in excel.

Click here for file

[http://www.biomedcentral.com/content/supplementary/14712164-6-180-S2.doc]

\section{Acknowledgements}

We would like to thank Dr. H. McDermid from University of Alberta, Canada for providing a cell line carrying a duplication of the 22q I 1.2 region for this study [25]. We would like to recognize the invaluable input from Dr. I. Teshima and for their technical assistance the researchers at The Hospital for Sick Children, Toronto, Canada and also Dr. Sandy Der's lab at The University of Toronto. Also we would like to thank Dr. G. Underwood (Bioproperties, Australia) Dr. C. Jones (CAB International, UK) and Dr. A. Moody (Astra Zeneca, UK) for their critical review of this manuscript. This research was supported by the National Cancer Institute of Canada with funds from the Canadian Cancer Society.

\section{References}

I. Ishkanian AS, Malloff CA, Watson SK, DeLeeuw RJ, Chi B, Coe BP, Snijders A, Albertson DG, Pinkel D, Marra MA, Ling V, MacAulay C, Lam WL: A tiling resolution DNA microarray with complete coverage of the human genome. Nat Genet 2004, 36:299-303.

2. Koolen DA, Vissers LE, Nillesen W, Smeets D, van Ravenswaaij CM, Sistermans EA, Veltman JA, de Vries BB: A novel microdeletion, $\operatorname{del}(2)(q 22.3 q 23.3)$ in a mentally retarded patient, detected by array-based comparative genomic hybridization. Clin Genet 2004, 65:429-432.

3. Shaw-Smith C, Redon R, Rickman L, Rio M, Willatt L, Fiegler H, Firth H, Sanlaville D, Winter R, Colleaux L, Bobrow M, Carter NP: Microarray based comparative genomic hybridisation (arrayCGH) detects submicroscopic chromosomal deletions and duplications in patients with learning disability/mental retardation and dysmorphic features. J Med Genet 2004, 4I:24I-248.

4. Babovic-Vuksanovic D, Jenkins SC, Ensenauer R, Newman DC, Jalal $S M$ : Subtelomeric deletion of $18 \mathrm{p}$ in an adult with paranoid schizophrenia and mental retardation. Am J Med Genet A 2004, I 24:318-322.

5. Mantripragada KK, Buckley PG, de StahI TD, Dumanski JP: Genomic microarrays in the spotlight. Trends Genet 2004, 20:87-94.

6. Mantripragada KK, Tapia-Paez I, Blennow E, Nilsson P, Wedell A, Dumanski JP: DNA copy-number analysis of the 22q I deletion-syndrome region using array-CGH with genomic and PCR-based targets. Int J Mol Med 2004, 13:273-279.

7. Saitta SC, Harris SE, Gaeth AP, Driscoll DA, McDonald-McGinn DM, Maisenbacher MK, Yersak JM, Chakraborty PK, Hacker AM, Zackai $\mathrm{EH}$, Ashley T, Emanuel BS: Aberrant interchromosomal exchanges are the predominant cause of the $22 \mathrm{qII} .2$ deletion. Hum Mol Genet 2004, 13:417-428.

8. Kurahashi H, Tsuda E, Kohama R, Nakayama T, Masuno M, Imaizumi K, Kamiya T, Sano T, Okada S, Nishisho I: Another critical region for deletion of 22q I : a study of 100 patients. Am J Med Genet 1997, 72:180-185.

9. Amati F, Conti E, Novelli A, Bengala M, Diglio MC, Marino B, Giannotti A, Gabrielli O, Novelli G, Dallapiccola B: Atypical deletions suggest five 22q I I.2 critical regions related to the DiGeorge/ velo-cardio-facial syndrome. Eur J Hum Genet 1999, 7:903-909.

10. Lu JH, Chung MY, Betau H, Chien HP, Lu JK: Molecular characterization of tetralogy of fallot within Digeorge critical region of the chromosome 22. Pediatr Cardiol 200I, 22:279-284.

11. Rauch A, Pfeiffer RA, Leipold G, Singer H, Tigges M, Hofbeck M: A novel 22q I 1.2 microdeletion in DiGeorge syndrome. Am J Hum Genet 1999, 64:659-666.

12. Saitta SC, McGrath JM, Mensch H, Shaikh TH, Zackai EH, Emanuel BS: A 22q I I.2 deletion that excludes UFDIL and CDC45L in a patient with conotruncal and craniofacial defects. Am J Hum Genet 1999, 65:562-566.

13. Ensenauer RE, Adeyinka A, Flynn HC, Michels VV, Lindor NM, Dawson DB, Thorland EC, Lorentz CP, Goldstein JL, McDonald MT, Smith WE, Simon-Fayard E, Alexander AA, Kulharya AS, Ketterling RP, Clark RD, Jalal SM: Microduplication 22q I I.2, an emerging syndrome: clinical, cytogenetic, and molecular analysis of thirteen patients. Am J Hum Genet 2003, 73: $1027-1040$.

14. Ikeuchi T: Extending the capabilities of human chromosome analysis: from high-resolution banding to chromatin fiberFISH. Hum Cell 1997, 10:121-134.

15. Sellner LN, Taylor GR: MLPA and MAPH: new techniques for detection of gene deletions. Hum Mutat 2004, 23:4|3-4|9.

16. UCSC Genome Bioinformatics [http://genome.ucsc.edu/]

17. NCBI [http://www.ncbi.nlm.nih.gov/]

18. Roe Lab Human Genomic DNA Sequencing Progress [http:/ /www.genome.ou.edu/hum totals.html]

19. Egenome [http://egenome.chop.edu/]

20. Human BAC Resource [http://www.ncbi.nlm.nih.gov/genome/ cyto/hbrc.shtml]

21. Human Organized Whole Genome Database [http://wwwalis.tokyo.jst.go.jp/HOWDY/]

22. YACs on Chromosome 22 [http://www.biologia.uniba.it/rmc/2YAC-BAC/YAC/YAC-22.html]

23. Genatlas [http://www.dsi.univ-paris5.fr/genatlas/] 
24. Human Genome Segmental Duplication Database [http:// projects.tcag.ca/humandup/]

25. Yobb TM, Somerville MJ, Willatt L, Firth HV, Harrison K, Mackenzie J, Gallo N, Morrow BE, Shaffer LG, Babcock M, Chernos J, Bernier F, Sprysak K, Christiansen J, Haase S, Elyas B, Lilley M, Bamforth S, McDermid HE: Microduplication and triplication of 22q I I.2: a highly variable syndrome. Am J Hum Genet 2005, 76:865-876.

26. Carlson C, Sirotkin H, Pandita R, Goldberg R, McKie J, Wadey R, Patanjali SR, Weissman SM, Anyane-Yeboa K, Warburton D, Scambler P, Shprintzen R, Kucherlapati R, Morrow BE: Molecular definition of $22 q I I$ deletions in $I 5 I$ velo-cardio-facial syndrome patients. Am J Hum Genet 1997, 61:620-629.

27. Demczuk S, Aledo R, Zucman J, Delattre O, Desmaze C, Dauphinot L, Jalbert P, Rouleau GA, Thomas G, Aurias A: Cloning of a balanced translocation breakpoint in the DiGeorge syndrome critical region and isolation of a novel potential adhesion receptor gene in its vicinity. Hum Mol Genet 1995, 4:55I-558.

28. Funke B, Edelmann L, McCain N, Pandita RK, Ferreira J, Merscher S, Zohouri M, Cannizzaro L, Shanske A, Morrow BE: Der(22) syndrome and velo-cardio-facial syndrome/DiGeorge syndrome share a I.5-Mb region of overlap on chromosome $22 \mathrm{qI}$. Am J Hum Genet 1999, 64:747-758.

29. Kurahashi H, Akagi K, Inazawa J, Ohta T, Niikawa N, Kayatani F, Sano $\mathrm{T}$, Okada S, Nishisho I: Isolation and characterization of a nove gene deleted in DiGeorge syndrome. Hum Mol Genet 1995, 4:54I-549.

30. Lindsay EA: Chromosomal microdeletions: dissecting del22q I I syndrome. Nat Rev Genet 200I, 2:858-868.

31. Lindsay EA, Vitelli F, Su H, Morishima M, Huynh T, Pramparo T, Jurecic V, Ogunrinu G, Sutherland HF, Scambler PJ, Bradley A, Baldini A: TbxI haploinsufficieny in the DiGeorge syndrome region causes aortic arch defects in mice. Nature 200I, 4I 0:97-I0I.

32. McQuade L, Christodoulou J, Budarf M, Sachder R, Wilson M, Emanuel B, Colley A: Patient with a 22q I I.2 deletion with no overlap of the minimal DiGeorge syndrome critical region (MDGCR). Am J Med Genet 1999, 86:27-33.

33. Perez E, Sullivan KE: Chromosome 22q I I.2 deletion syndrome (DiGeorge and velocardiofacial syndromes). Curr Opin Pediatr 2002, 1 4:678-683.

34. Shaikh TH, Kurahashi H, Saitta SC, O'Hare AM, Hu P, Roe BA, Driscoll DA, McDonald-McGinn DM, Zackai EH, Budarf ML, Emanuel BS: Chromosome 22-specific low copy repeats and the 22q II.2 deletion syndrome: genomic organization and deletion endpoint analysis. Hum Mol Genet 2000, 9:489-50I.

35. Johnson A Minoshima S, Asakawa S, Shimizu N, Shizuya H, Roe BA, McDermid HE: A I.5-Mb contig within the cat eye syndrome critical region at human chromosome 22qli.2. Genomics 1999, 57:306-309.

36. Kent WJ: BLAT--the BLAST-like alignment tool. Genome Res 2002, I 2:656-664.

37. Radonic A, Thulke S, Mackay IM, Landt O, Siegert W, Nitsche A: Guideline to reference gene selection for quantitative realtime PCR. Biochem Biophys Res Commun 2004, 3 I 3:856-862.

38. Altschul SF, Gish W, Miller W, Myers EW, Lipman DJ: Basic local alignment search tool. J Mol Biol 1990, 215:403-410.

39. Meijerink J, Mandigers C, van de Locht L, Tonnissen E, Goodsaid F, Raemaekers J: A novel method to compensate for different amplification efficiencies between patient DNA samples in quantitative real-time PCR. J Mol Diagn 200I, 3:55-6I.

40. Tichopad A, Dilger M, Schwarz G, Pfaffl MW: Standardized determination of real-time PCR efficiency from a single reaction set-up. Nucleic Acids Res 2003, 3 I:e I 22.

41. Moody A, Sellers S, Bumstead N: Measuring infectious bursal disease virus RNA in blood by multiplex real-time quantitative RT-PCR. J Virol Methods 2000, 85:55-64.

42. Sijben JW, Klasing KC, Schrama JW, Parmentier HK, van der Poel JJ, Savelkoul HF, Kaiser P: Early in vivo cytokine genes expression in chickens after challenge with Salmonella typhimurium lipopolysaccharide and modulation by dietary $n--3$ polyunsaturated fatty acids. Dev Comp Immunol 2003, 27:6 II-6I9.

43. Parr C, Jiang WG: Quantitative analysis of lymphangiogenic markers in human colorectal cancer. Int J Oncol 2003, 23:533-539.

44. Cairns JP, Chiang PW, Ramamoorthy S, Kurnit DM, Sidransky D: A comparison between microsatellite and quantitative PCR analyses to detect frequent p 16 copy number changes in primary bladder tumors. Clin Cancer Res 1998, 4:44I-444.

45. Ivanov D, Kirov G, Norton N, Williams HJ, Williams NM, Nikolov I, Tzwetkova R, Stambolova SM, Murphy KC, Toncheva D, Thapar A, O'Donovan MC, Owen MJ: Chromosome 22q I I deletions, velocardio-facial syndrome and early-onset psychosis. Molecular genetic study. $\mathrm{Br} /$ Psychiatry 2003, I 83:409-4 I3.

46. Kariyazono $\mathrm{H}$, Ohno $\mathrm{T}$, Ihara $\mathrm{K}$, Igarashi $\mathrm{H}$, Joh-o K, Ishikawa S, Hara $\mathrm{T}$ : Rapid detection of the $22 \mathrm{q}$ I I.2 deletion with quantitative real-time PCR. Mol Cell Probes 200I, 15:7I-73.

47. Shi YR, Hsieh KS, Wu JY, Lee CC, Tsai CH, Yu MT, Chang JS, Tsai FJ: Genetic analysis of chromosome 22q I 1.2 markers in congenital heart disease. J Clin Lab Anal 2003, 17:28-35.

48. Nicholas C. Dracopoli, Jonathan L. Haines, Bruce R. Korf, Cynthia C. Morton, Christine E. Seidman, J.G. Seidman, Smith DR: Short Protocols in Human Genetics. Edited by: Leonard SA. , John Wiley \& Sons, Inc.; 1994.

Publish with Biomed Central and every scientist can read your work free of charge

"BioMed Central will be the most significant development for disseminating the results of biomedical research in our lifetime. "

Sir Paul Nurse, Cancer Research UK

Your research papers will be:

- available free of charge to the entire biomedical community

- peer reviewed and published immediately upon acceptance

- cited in PubMed and archived on PubMed Central

- yours - you keep the copyright
BiolMedcentral 